\title{
Nanocavity clock spectroscopy: resolving competing exciton dynamics in $\mathrm{WSe}_{2} / \mathrm{MoSe}_{2}$ heterobilayers
}

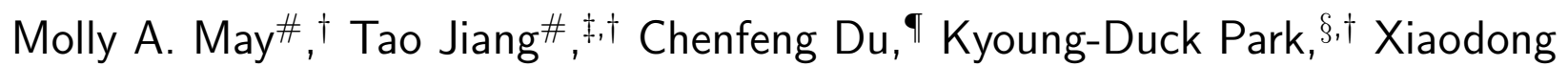
Xu, Alexey Belyanin, ${ }^{*}, \|$ and Markus B. Raschke, $\dagger$

$\dagger$ Department of Physics, Department of Chemistry, and JILA, University of Colorado, Boulder, CO 80309

$\ddagger M O E$ Key Laboratory of Advanced Micro-Structured Materials, and School of Physics Science and Engineering, Tongji University, Shanghai 200092, China

TDepartment of Physics, University of Washington, Seattle, WA 98195

$\S$ Department of Physics, School of Natural Sciences, Ulsan National Institute of Science and Technology, Ulsan, South Korea

|lDepartment of Physics and Astronomy, Texas A\&3M University, College Station, Texas 77843

E-mail: belyanin@tamu.edu(A.B.); markus.raschke@colorado.edu(M.B.R.)

\section{Supporting Information Available}

\section{Tip-enhanced photoluminescence spectroscopy}

In the TEPL spectroscopy setup, the sample is mounted to a piezoelectric transducer (PZT, P-611.3, Physik Instrumente) with sub-nm precision positioning with respect to a mechani- 
cally driven and electrically controlled quartz tuning fork (resonance frequency $=32 \mathrm{kHz}$ ), which was used to regulate the tip-sample distance using the AFM shear-force frequency feedback. Electrochemically etched Au tips ( $\sim 5-10 \mathrm{~nm}$ apex radius) were attached to the tuning fork and coarsely positioned using a stepper motor (MX25, Mechonics AG), and shearforce feedback and sample positioning were controlled by a digital AFM controller (R9, RHK Technology). The sample was mounted at a $35^{\circ}$ angle with respect to the tip axis to maximize the plasmonic field confinement as described previously ${ }^{1}$. Excitation light was focused onto the tip-sample interface using an objective lens (NA=0.8, LMPLFLN100 $\times$, Olympus) with a half wave plate for polarization control. Excitation was provided by a helium-neon laser beam $(632.8 \mathrm{~nm}, \leq 1 \mathrm{~mW})$ at a fluence of $\leq 10^{7} \mathrm{~W} / \mathrm{cm}^{2}$, and the photoluminescence (PL) signal was monitored for stability to eliminate the possibility of laser-induced sample damage. The tip-scattered near-field PL signal was collected in a backscattering geometry, passed through a dichroic mirror with a $633 \mathrm{~nm}$ cut-off and detected with a spectrometer (f $=500 \mathrm{~mm}$, SpectraPro 500i, Princeton Instruments) with a thermoelectrically cooled, electron-multiplied, charge-coupled device (CCD, ProEM+: 1600 eXcelon3, Princeton Instruments). The spectrometer was calibrated using a hydrogen mercury lamp and a 150 $\mathrm{g} / \mathrm{mm}$ grating blazed for $800 \mathrm{~nm}$ is used to provide high bandwidth spectral information.

Examples of near-field (blue) and far-field (red) PL spectra are also included in Fig. 1 (a), with the far-field PL measured when the tip-sample distance is much larger than the near-field interaction regime. Because the $\mathrm{Au}$ plasmonic tip is tilted $35^{\circ}$ with respect to the sample surface normal, it provides nearly equal in-plane and out-of-plane near-field enhancement. Thus, significant near-field enhancement of both the IX PL with in-plane and out-of-plane dipole moments and the $\mathrm{WSe}_{2}$ and $\mathrm{MoSe}_{2} \mathrm{PL}$ can be seen.

Several measurements were performed to confirm that the heterobilayer was not damaged by the interaction with the AFM probe. First, we measured the hysteresis of the optical signal upon approach (dark blue) and retraction (light blue) of the tip as shown in Figure 1 (b). This revealed smooth, continuous behavior in the contact region and a return to the 
initial PL intensity after contact. As damage to the 2D material lattice generally leads to a discontinuity in the PL amplitude due to the generation of defect states and significant enhancement of the tip plasmon as it contacts the gold substrate, this smooth, reproducible evolution of the PL indicates that the sample remained undamaged during the measurement.

Additionally, the presence of strain in WSe2 and MoSe2 causes a spectral shift of their exciton resonances. Here, we observed no measurable spectral shift during contact, which further confirms that the tip did not cause significant strain in the crystal lattice.

Finally, any significant force interaction between the soft gold AFM tip and the 2D material layers is likely to cause damage to the tip which would lead to unstable AFM feedback. The smooth hysteresis of the shear force feedback amplitude shown in Figure 1 (b) (red) indicates that contact with the sample did not enter an unstable or high force regime at any time during the approach or retraction of the AFM tip.
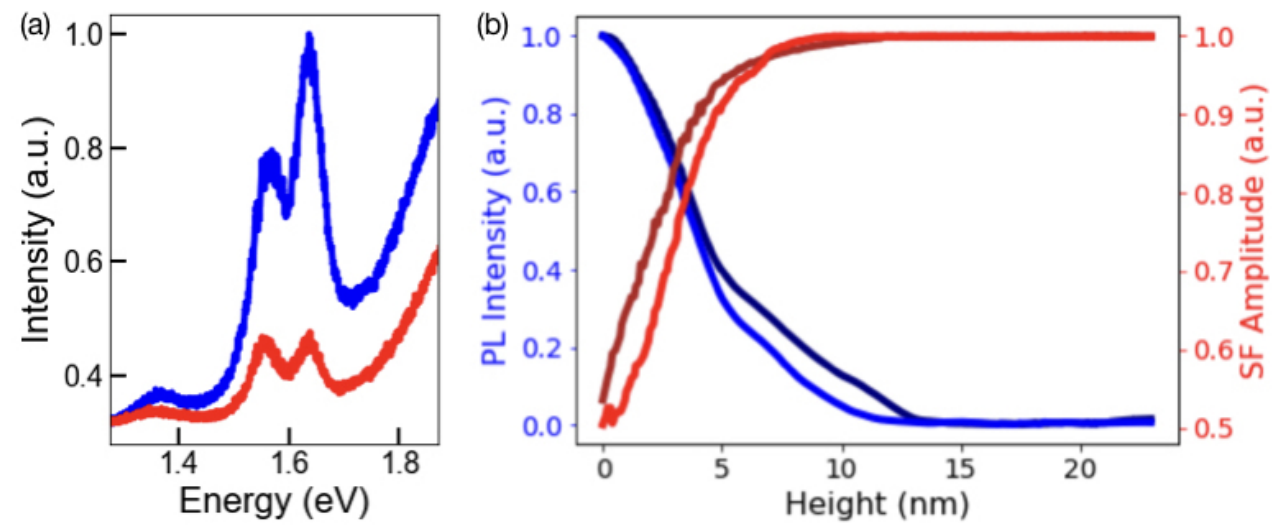

Figure 1: (a) Examples of near-field (blue) and far-field (red) PL spectra showing significant enhancement of all excitons. (b) Hysteresis of the near-field optical signal (blue) and SF feedback amplitude (red) on approach (dark) and retract (light).

\section{Coupled Rate Equations}

In order to quantify the competing radiative and nonradiative processes underlying our observations, we develop a rate equation model to describe the distance dependence of the different excitonic signals. We treat the tip-substrate junction as a plasmonic cavity in 
which the incident laser field $E_{0}$ experiences near-field enhancement. The excitation rate for the intralayer excitons $F_{X}$ is then proportional to the cavity enhanced field $F_{X} \propto\left|E / E_{0}\right|^{2}$, where $X=M$ or $W$ stand for the $\mathrm{MoSe}_{2}$ or $\mathrm{WSe}_{2}$ intralayer exciton species. This near-field enhanced excitation rate scales as $F_{X} \propto(R / z)^{m}$ where $\mathrm{R}$ is the tip radius, $\mathrm{z}$ is the distance between the tip and the Au substrate, and $m$ determines a geometry related growth rate. This near-field enhanced excitation dominates over the long range emission behavior for $z>5 \mathrm{~nm}$. In contrast, the IX is populated by charge transfer from the intralayer exciton population.

The total decay rate of each intralayer exciton species is the sum of Purcell-controlled radiative decay into the cavity near-field $\Gamma_{X}^{r a d}$, extrinsic tip-induced nonradiative recombination $\Gamma_{X}^{n r a d}$, and the to first order tip-independent nonradiative recombination rates for each exciton intrinsic to the material. We assume that the tip-independent contribution is dominated by ultrafast interlayer charge transfer, which we use as a reference clock with rate $\Gamma_{\mathrm{CT}}=44 \mathrm{fs}$ based on previous measurements as discussed in section $\mathrm{IV}^{2-8}$.

The excitation of the intralayer excitons $N_{X}$ and interlayer excitons $N_{I X}$ populations is then given by the differences between the different excitation and relaxation rates:

$$
\begin{aligned}
\frac{d N_{X}}{d t} & =F_{X}-\left(\Gamma_{X}^{r a d}+\Gamma_{X}^{n r a d}+\Gamma_{\mathrm{CT}}\right) N_{X}, \\
\frac{d N_{I X}}{d t} & =\Gamma_{C T}\left(N_{M}+N_{W}\right)-\left(\Gamma_{I X}^{r a d}+\Gamma_{I X}^{n r a d}\right) N_{I X} .
\end{aligned}
$$

Here, the radiative decay rate for each species is affected by the Purcell enhancement $F_{P}$ of the cavity $\Gamma_{X, I X}^{r a d} \propto Q \frac{\lambda^{3}}{V_{c}}$ where $Q$ is the cavity quality factor, $\lambda$ is the emission wavelength, and $V_{c}$ is the z-dependent cavity volume ${ }^{9,10}$. The cavity volume is assumed to decrease as a power law with the tip-sample distance, yielding $\Gamma_{X, I X}^{r a d}=\Gamma_{X, I X}^{r a d, 0}\left(F_{P}+1\right) \propto(z+d)^{-n}+\Gamma_{X, I X}^{r a d, 0}$ where $d$ is the minimum tip-substrate separation, $n$ is the scaling exponent, and $\Gamma_{X, I X}^{r a d, 0}$ is the radiative decay rate into free space.

Similarly, the tip-induced nonradiative exciton recombination rate for each species is given 
by dipole-dipole coupling between exciton and metal ${ }^{11}$, which can be modeled as $\Gamma_{X, I X}^{n r a d} \propto$ $(R /(z+\delta z))^{l}+\Gamma_{X, I X}^{n r a d, 0}$ where $l$ is a scaling exponent, $\Gamma_{X, I X}^{n r a d, 0}$ is the intrinsic, unperturbed nonradiative exciton decay, and $\delta z$ is the minimum distance between the exciton dipole and the tip material.

These coupled rate equations can be solved analytically in the steady state to find the distance dependent populations of each exciton species,

$$
\begin{array}{r}
N_{X}=\frac{F_{X}}{\Gamma_{X}^{r a d}+\Gamma_{X}^{n r a d}+\Gamma_{\mathrm{CT}}}, \\
N_{I X}=\frac{\Gamma_{C T}}{\Gamma_{I X}^{r a d}+\Gamma_{I X}^{n r a d}}\left(N_{M}+N_{W}\right)
\end{array}
$$

where subscript $X$ stands for $M$ or $W$. The PL intensity for each species is then given by

$$
P_{X, I X}=\eta \Gamma_{X, I X}^{r a d} N_{X, I X}
$$

where the outcoupling efficiency of the cavity near-field radiation into far-field is represented by $\eta$. If the cavity losses are mainly due to radiation outcoupled into the far-field, $\eta$ is closely related to the inverse cavity quality factor $\eta \sim 1 / Q$ such that this term cancels with the factor of $Q$ from the radiative decay term. However, it is also possible that the cavity losses are nonradiative due to photon absorption.

Several assumptions were made in this model, and while they are overall quite robust, there are some limiting cases where they break down. For example, the assumption that the excitation rate $\mathrm{Fx}$ is proportional to the intracavity field intensity is valid as long as the intensity is well below saturation. The latter is true under our experimental conditions, and the PL excitation did not show any signs of saturation upon approach. Furthermore, the separation of the total decay rate of each exciton species into radiative and nonradiative components in the second part of Equation 1 requires no approximation, and the functional forms assumed for each term are quite general. They assume only that the z-dependent 
parts of the radiative and nonradiative decay increase proportionally to the cavity density of states and the dipole-dipole coupling to the tip, respectively, which are both well-established theoretically. Furthermore, the interlayer exciton has both in-plane and out-of-plane dipole orientation components. These contribute to the relaxation rates with the same tip-sample distance scaling but different weights; moreover, their relative contribution may depend on the tip-sample distance. However, our measurements are not sensitive enough to separate these relative contributions. Finally, we neglected exciton-exciton interactions, which could make some of the decay rates exciton density-dependent. This is a reasonable assumption: for an excitation power below $1 \mathrm{~mW}$, ultrafast nonradiative decay rates below $1 \mathrm{ps}$, and low absorbance 1-2\% the steady-state density of excitons is very low: below $10^{10} \mathrm{~cm}^{-2}$.

\section{Scaling of PL Intensity}

One can distinguish between two limiting dynamic regimes of the exciton PL. When $\Gamma_{C T} \gg$ $\Gamma_{X, I X}^{n r a d}$ which is the case for large $z$, the asymptotic formulas for the PL intensity are

$$
\begin{aligned}
P_{X} & \approx \frac{\Gamma_{X}^{r a d} \eta F_{X}}{\Gamma_{C T}} \\
P_{I X} & \approx \frac{\Gamma_{I X}^{r a d} \eta}{\Gamma_{I X}^{n r a d}}\left(F_{M}+F_{W}\right) .
\end{aligned}
$$

These predict a rapid growth of $P_{M, W}$ with $z$ and a somewhat slower growth of $P_{I X}$ because it has an extra factor $\Gamma_{I X}^{n r a d}$ in the denominator which grows with $z$.

In the opposite limit of small $z$ the nonradiative decay becomes much faster than $\Gamma_{C T}$, in which case

$$
\begin{aligned}
P_{X} & \approx \frac{\Gamma_{X}^{r a d} \eta F_{X}}{\Gamma_{X}^{n r a d}} \\
P_{I X} & \approx \frac{\Gamma_{C T} \Gamma_{I X}^{r a d} \eta}{\Gamma_{I X}^{n r a d}}\left(\frac{F_{M}}{\Gamma_{M}^{n r a d}}+\frac{F_{W}}{\Gamma_{W}^{n r a d}}\right) .
\end{aligned}
$$


Note that in both limiting cases the nonradiative decay rates $\Gamma_{X, I X}^{n r a d}$ cancel out from the expressions for the ratios of PL intensity from different exciton species. Therefore, measurements at fixed $z$ would be insensitive to the values of this parameter. Only by going through a crossover from large $z$ to small $z$ regimes one is able to retrieve the most complete set of parameters.

Finally, note that the near-field enhancement term is expected to grow as $1 / z^{6}$ at long distance where the cavity geometry is similar to a point dipole above a plane, and as $1 / z^{2}$ at short range where the cavity geometry is similar to a capacitor. Thus, the fitted power-law exponent of $m=(2.5 \pm 0.1)$ indicates that we are near the short range limit. Furthermore, the overlap of the exciton dipole and the tip material, which determines the nonradiative damping rate to the tip, is expected to grow as $1 / z^{3}$ for a flat tip geometry and faster for the actual surface which is convex to the sample, which is consistent with our measured exponent $l=(3.7 \pm 0.1)$.

\section{Details of the Charge Transfer Time}

Most existing measurements of the charge transfer time have been limited in their temporal resolution by their instrument response time and thus provide only an upper limit of the true $\Gamma_{C T}{ }^{2-8}$. While several of these measurements set upper bounds on the few hundred femtosecond level, this does not rule out the possibility of a faster charge transfer time. For this reason, we focused on the results of studies with the fastest temporal resolution in refs. [26]-[30] to best estimate CT time. The results of these studies were averaged to determine the charge transfer time of 44 fs that was used in the main text of the paper. However, due to the importance of this point for our analysis, and the significant remaining uncertainty in reported literature values, we have also performed the analysis assuming a $100 \mathrm{fs}$ charge transfer time for comparison. The resulting lifetimes are provided in table 1. 
Table 1: Fitted exciton lifetimes assuming $\Gamma_{C T}=100 \mathrm{fs}$ and $\Gamma_{C T}=44 \mathrm{fs}$.

\begin{tabular}{|l|l|l|}
\hline Parameter & Value $\left(\Gamma_{C T}=100 \mathrm{fs}\right)$ & Value $\left(\Gamma_{C T}=44 \mathrm{fs}\right)$ \\
\hline$\tau_{I X}^{\text {rad,0 }}$ & $(213 \pm 61) \mathrm{ns}$ & $(94 \pm 27) \mathrm{ns}$ \\
\hline$\tau_{I X}^{\text {nrad }, 0}$ & $(1.36 \pm 0.45) \mathrm{ps}$ & $(0.6 \pm 0.2) \mathrm{ps}$ \\
\hline$\tau_{X}^{\text {rad,0 }}$ & $(1.59 \pm 0.45) \mathrm{ns}$ & $(0.7 \pm 0.2) \mathrm{ns}$ \\
\hline$\tau_{X}^{\text {nrad }, 0}$ & $(5.9 \pm 1.6) \mathrm{ps}$ & $(2.6 \pm 0.7) \mathrm{ps}$ \\
\hline
\end{tabular}




\section{References}

(1) Park, K.-D.; Raschke, M. B. Polarization Control with Plasmonic Antenna Tips: A Universal Approach to Optical Nanocrystallography and Vector-Field Imaging. Nano Letters 2018, 18, 2912-2917.

(2) Ceballos, F.; Bellus, M. Z.; Chiu, H.-Y.; Zhao, H. Ultrafast Charge Separation and Indirect Exciton Formation in a $\mathrm{MoS}_{2}-\mathrm{MoSe}_{2}$ van der Waals Heterostructure. ACS Nano 2014, 8, 12717-12724.

(3) Ji, Z.; Hong, H.; Zhang, J.; Zhang, Q.; Huang, W.; Cao, T.; Qiao, R.; Liu, C.; Liang, J.; Jin, C.; Jiao, L.; Shi, K.; Meng, S.; Liu, K. Robust Stacking-Independent Ultrafast Charge Transfer in $\mathrm{MoS}_{2} / \mathrm{WS}_{2}$ Bilayers. ACS Nano 2017, 11, 12020-12026.

(4) Peng, B.; Yu, G.; Liu, X.; Liu, B.; Liang, X.; Bi, L.; Deng, L.; Sum, T. C.; Loh, K. P. Ultrafast charge transfer in $\mathrm{MoS}_{2} / \mathrm{WSe}_{2} \mathrm{p}-\mathrm{n}$ Heterojunction. 2D Materials 2016, 3, 025020 .

(5) Zheng, Q.; Xie, Y.; Lan, Z.; Prezhdo, O. V.; Saidi, W. A.; Zhao, J. Phonon-coupled ultrafast interlayer charge oscillation at van der Waals heterostructure interfaces. Physical Review B 2018, 97, 205417.

(6) Zhu, H.; Wang, J.; Gong, Z.; Kim, Y. D.; Hone, J.; Zhu, X.-Y. Interfacial Charge Transfer Circumventing Momentum Mismatch at Two-Dimensional van der Waals Heterojunctions. Nano Letters 2017, 17, 3591-3598.

(7) Chen, H.; Wen, X.; Zhang, J.; Wu, T.; Gong, Y.; Zhang, X.; Yuan, J.; Yi, C.; Lou, J.; Ajayan, P. M.; Zhuang, W.; Zhang, G.; Zheng, J. Ultrafast formation of interlayer hot excitons in atomically thin MoS2/WS2 heterostructures. Nature Communications 2016, 7, 12512. 
(8) Hong, X.; Kim, J.; Shi, S.-F.; Zhang, Y.; Jin, C.; Sun, Y.; Tongay, S.; Wu, J.; Zhang, Y.; Wang, F. Ultrafast charge transfer in atomically thin MoS2/WS2 heterostructures. Nature Nanotechnology 2014, 9, 682-686.

(9) Park, K.-D.; May, M. A.; Leng, H.; Wang, J.; Kropp, J. A.; Gougousi, T.; Pelton, M.; Raschke, M. B. Tip-enhanced strong coupling spectroscopy, imaging, and control of a single quantum emitter. SCIENCE ADVANCES 2019, 8.

(10) May, M. A.; Fialkow, D.; Wu, T.; Park, K.; Leng, H.; Kropp, J. A.; Gougousi, T.; Lalanne, P.; Pelton, M.; Raschke, M. B. Nano-Cavity QED with Tunable Nano-Tip Interaction. Advanced Quantum Technologies 2020, 3, 1900087.

(11) Forster, T. Intermolecular Energy Migration and Fluorescence. Annals Phys. 1948, 2, $55-75$. 